\title{
Las ciencias cognitivas: preámbulo para comprender el funcionamiento de los esquemas del ser bumano
}

\author{
Dr. Álvaro Antonio Escobar Soriano \\ Educación e Intervención Social \\ UNAN-Managua \\ aescobar@unan.edu.ni / alvess007@gmail.com
} https://doi.org/10.5377/torreon.v7i19.7908

Palabras clave: ciencias cognitivas, autopoiesis, plasticidad cerebral, neuronas espejo, memoria, holograma nervioso.

\section{RESUMEN}

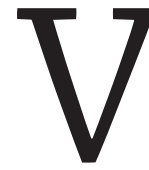

arias generaciones de estudios delas ciencias cognitivas, han dado como resultado una amplia cantidad de teorías. En estas, se abordan diferentes aspectos del sistema que permiten al ser humano funcionar en diferentes ambientes. Esto es posible, porque el sistema cerebral es plástico (adaptable), se reestructura a sí mismo en una escala de la realidad, imperceptible para los sentidos humanos. A la par de esta cualidad, existen otras que codifican información. Ese es el caso del sistema neuronal espejo que codifica las intenciones de los demás, lo que permite entendernos.

Los esquemas de aprendizaje se pueden estructurar, considerando el modelo holográfico de la mente, que expone la manera cómo la conciencia se distribuye por todo el sistema cerebral y funciona como un mecanismo de recuperación de información desde las diferentes áreas. Estos sistemas tienen como producto lo que en neurociencia se denomina memoria, la que es individual y colectiva.

Finalmente, el lenguaje sustenta estas capacidades mentales y permite la manifestación comunicativa de los esquemas, a través de la interacción con los demás. 


\section{INTRODUCCIÓN}

Las ideas desarrolladas en este artículo teórico provienen de varias generaciones de estudios de las ciencias cognitivas, pero conducidas por una perspectiva integradora, que ayuda a tener una visión general sobre cómo funciona el ser humano ${ }^{1}$.

Por lo planteado en el párrafo anterior, se afirma que el hombre como especie es bio-neuropsicológico, comunicativo y sociocultural. Todos estos conceptos se resumen sencillamente en que es un ser humano, vive en la realidad -en un contexto-ambiente / en un espacio-tiempo- y actúa para transformarla.

Desde el punto de vista biológico, siguiendo a Maturana (1990) el ser humano, es un ser vivo autopoiético de segundo orden (la célula es un ser vivo de primer orden), que se desarrolla en un ambiente social. Por consiguiente, los fenómenos sociales pertenecen a la categoría de los fenómenos biológicos, en tanto en cuanto, se producen por el actuar de seres que implican autopoiesis. Este concepto define al proceso con el cual un organismo vivo, mediante una red autónoma, se produce a sí mismo en su dinámica cerrada (Maturana y Pörksen, 2004). Esta manera de comprender lo vivo, en especial el sistema nervioso, la desarrolló Maturana desde 1968:

(...) había comprendido que los fenómenos asociados a la percepción se entendían sólo si se entendía el operar del sistema nervioso como una red circular cerrada de correlaciones internas, y simultáneamente entendía que la organización del ser vivo se explicaba a sí misma al verla como un operar circular cerrado de producción de componentes que producían la misma red de relaciones de componentes que los generaba (teoría que posteriormente denominó autopoiesis), (Maturana y Varela, 2003, p. XXI).

El fenómeno viviente permite a este autor, proponer una serie de leyes sistemáticas básicas con las cuales se amplía la consciencia de que cada sujeto es bilógico-cultural, genera y evoca los mundos que habita en su diario convivir (Maturana y Dávila, 2008) en su comunidad, institución laboral o centro de estudios. Este sistema de leyes tiene implicaciones que abarcan todos los ámbitos de la vida humana, vistas como posibilidad de:

1. La primera generación en ciencias de la cognición sostiene una propuesta teórica centrada en los conceptos de representación y computación como forma humana de procesamiento de la información. La segunda desarrolla el modelo conexionista, con el cual el ser humano construye la realidad mediante redes de sentido que se activan a nivel neuronal. La tercera construye su propuesta teórica sobre una base corporal, interactiva y situacional de la manera en que el ser humano construye su realidad. La última versión -toma aspectos de las generaciones anteriores- se basa en el enfoque experiencialista y en el principio de que toda experiencia es compleja con diversos aspectos/ niveles de jerarquía, interrelacionados y conjugados en una matriz sociocultural que favorece: el desarrollo de las capacidades de su sistema nervioso, el establecimiento de asociaciones significativas entre situaciones diversas, la valoración de los procesos emergentes que surgen de la práctica socio-cultural y dotan de sistematicidad la cognición humana en forma de redes de sentido compartido; las representaciones de determinados aspectos de su experiencia a través de otras prácticas y la transformación de sus prácticas socio-culturales al construir y emplear artefactos, finalmente mediante el lenguaje el estudio de la creatividad, de la capacidad de estandarización de procesos cognitivos relacionados directamente con el significado y el uso de instrumentos/artefactos del entorno (Redondo, 2012). 
- Conocer, entender y explicar.

- Observar, reflexionar y comunicar a los demás, saca a la luz los cambios vividos, amplía la reflexión y la comprensión recursiva de sí mismo.

- Hacer, según regularidades y coherencias operacionales estructurales y funcionales del organismo vivo que se conservan en todas las circunstancias e instantes.

- Ampliar la capacidad recursiva de reflexionar sobre su propio vivir.

- Vivir la realidad, validarla, realizar distinciones sobre esta, aunque después se dude de su validez.

- Investigar la historia de constitución de la vida humana en su devenir.

- Cambiarse a sí mismo y al ambiente, manteniendo una relación equilibrada.

- Observar los dominios en los que existen y operan los seres vivos, en el cambiante presente continuo.

- Asimilar los cambios durante el proceso de transformación, permite la adaptación y conservación.

- Obtener resultados en relación con las posibilidades creadas en el devenir histórico.

- Operar como centro del ambiente-contexto donde se habita, es el reflejo de la matriz biológica de existencia.

- Obtener resultados con procesos que emplean dominios disjuntos de la realidad y del conocimiento.

Distinguir los componentes, la organización, funcionamiento, cambios y mantenimiento de la estructura (identidad) en un ser vivo, en tanto sistema cerrado y abierto al ambiente.

Todas las posibilidades permiten al ser humano vivir en una matriz biológico-cultural, que a su vez genera, realiza y conserva el nicho biológico-cultural en sus diferentes ámbitos socio-culturales: laboral, educativo, científico, artístico, deportivo, etc. Entonces, el resultado de este sistema es la síntesis matríztica, que se conoce como aprendizaje continuo. Luzoro (2006), considera que este ocurre "(...) cuando la conducta de un organismo varía durante su ontogenia de manera congruente con las variaciones del medio, y lo hace siguiendo un curso contingente a sus interacciones en él” (p.36). Esto es así, porque el organismo se adapta a ese medio particular (información, datos, significado), representándolo y almacenándolo en su memoria en forma de recuerdos. Durante este proceso conserva su organización corporal y mental como referencia que guía los cambios estructurales operados con el aprendizaje. 
Desde este doble punto de vista, el proceso de enseñanza-aprendizaje se reduce a la segunda parte del correlato, porque tanto el docente como sus discentes aportan, cada uno con su propia estructura mental, corporal y de conocimientos, a que se alcance un nuevo nivel de desarrollo cognitivo, social y cultural. En el aula de clase, todos se transforman, lo que se puede observar en la historia de sus interacciones: actuaciones y discursos.

\section{MATERIALES Y MÉTODOS}

Este artículo, es parte de la teoría que sustenta la tesis doctoral: "Esquemas de aprendizaje de la gramática". El principal método utilizado fue la búsqueda y rastreo de información, lo que se denomina método bibliográfico. De ahí que, De la Torre y Navarro (1982) expresen:

La adquisición u obtención del conocimiento, la fijación, organización y ampliación del mismo, así como su transmisión, requieren de normas especiales, de una metodología que precise y eduque en pensamiento y la expresión, que los estimulen y fortalezcan. Así pues, el método es un proceso lógico, surgido del raciocinio y de la inducción. (p. 3).

Considerando, que la obtención de la información ocurre de manera directa o indirecta, y este último procedimiento es común en la práctica investigativa actual (Sierra, 1986), el desarrollo de la temática: "Las ciencias cognitivas: preámbulo para comprender el funcionamiento de los esquemas del ser humano", centró su foco de selección en aquellos aspectos teóricos que permitieron crear la base para la comprensión cognitiva de las teorías que Piaget y los neopiagetianos desarrollaron.

Predominó en la indagación la conceptualización y la definición como estrategias que conforman el método interpretativo. Con este, se llegó de manera sistemática, a la estructuración de planteamientos teóricos, que favorecieron posteriormente la construcción de la teoría de esquemas la cual fue probada durante un proceso intenso de indagación en el aula de clase.

\section{LAS CIENCIAS COGNITIVAS PARA COMPRENDER EL FUNCIONAMIENTO DE LOS ESQUEMAS DE APRENDIZAJE HUMANO}

\subsection{La plasticidad neuronal}

Todas las distinciones que es capaz de realizar el ser humano en relación con el mundo interno y el externo, se pueden representar de manera adecuada y coherente a través del sistema perceptual de primer orden: vista, oído, tacto, olfato y gusto. Los conocimientos, pensamientos y sentimientos son esquemas estructurados en el cerebro mediante un proceso, que genera el sistema representacional primario o externo. Estos procesos pueden ser codificados en patrones de funcionamiento, según sea el perceptor empleado (Carrión, 2005). 
Sin embargo, es el sistema representacional de segundo orden (Sistema Nervioso Central), el que organiza y comanda las configuraciones que el primero ha ido formando. Esa organización no es estática, sino dinámica, flexible, porque tiende a cambiar y a reorganizarse a partir de la recurrente categorización realizada por este sistema, el cual está conformado por todos los esquemas de acción engramatizados en el cerebro.

Cada reconfiguración ocurre por la relación del ser humano con su ambiente y con otros miembros de su especie. Es decir, el lenguaje y la comunicación también moldean la estructura neuronal. Una serie de habilidades se involucran en el fenómeno comunicativo, Lugarini (1994) se refiere a ellas como "competencias del habla". Estas microhabilidades o microesquemas: ideativo, pragmático, sintáctico, semántico y técnico, comprenden numerosos elementos que a su vez son requisitos para que se cumpla la función comunicativa. Así, la búsqueda y manejo de información, los usos de la lengua, las maneras como se estructuran y funcionan sus esquemas paradigmáticos y sintagmáticos, la comprensión de lo expresado (hasta lo que se piensa), son coadyuvantes en la producción de unidades comunicativas, capaces de transferir mensajes hacia uno o varios oyentes, por consiguiente, capaces de transformar las redes neuronales y los esquemas que en ellas se forman (aprendizaje).

Por tanto, cualquier señal de interacción involucra una serie de procedimientos neurocognitivos, que funcionan de manera coordinada para que pueda producirsela comunicación humana. Todos los mensajes dirigidos al cerebro y provenientes de él son codificados en el lenguaje neuronal, y ese lenguaje es la activación del procesamiento inicial del sistema operativo de la mente, para recoger pequeñas y específicas partes del mundo exterior. Por ello, el cerebro es un potente filtro de información el cual es dirigido por esquemas mentales especializados de evaluación, lo cual no permite la acumulación caótica de información.

Esto es así, porque este sistema tiene una capacidad intrínseca denominada plasticidad cerebral, mediante la cual se reestructura a sí mismo en una escala de la realidad, imperceptible para los sentidos humanos. A esto es a lo que (Maturana y Varela, 2003; Maturana, 1990; Maturana y Pörksen, 2004; Maturana, en Luzoro, 2006 y Maturana y Dávila, 2008) denominan autopoiesis de organismos vivos, proceso que se produce en el sistema nervioso central del ser humano con todas sus implicaciones biológicas, neurológicas, psicológicas, socioculturales, etc.

La plasticidad cerebral es una propiedad del cerebro, en términos de causalidad, producida por el funcionamiento del sistema nervioso, los efectos del ambiente sobre el organismo vivo y la experiencia de este en relación con las situaciones a las que se enfrenta. Esta se define como la capacidad que tiene el sistema nervioso para cambiar, durante el desarrollo los circuitos neuronales. Se considera que el sistema de una persona adulta, también posee esa capacidad para aprender (nuevas habilidades, establecimiento de nuevos recuerdos, enfrentar lesiones 
cerebrales, enfrentar nuevas situaciones, etc.), la cual está basada en los cambios cuidadosamente regulados -breves o más duraderos según la experiencia- en las sinapsis neuronales (Purves et al, 2007).

La plasticidad, como capacidad del cerebro, generadora de aprendizaje fue corroborada por Kandell (2007) en diferentes experimentos con seres vivos llamados Aplysia. Este comprobó que la experiencia produce el aprendizaje y este es la síntesis final de procesos complejos de la maleabilidad neuronal:

- Tanto la anatomía del circuito neural como las modificaciones firmes que se producen en él son ejemplos de que la práctica implica perfección, a decir sustentan la memoria.

- Las modificaciones en las sinapsis interneuronales subyacentes al aprendizaje pueden ser suficientes para reconfigurar tanto la red neuronal como su capacidad de procesamiento de información.

- Formas distintas de aprendizaje pueden modificar las redes neuronales, atenuándola mediante habituación (memoria a largo plazo) o reforzándola a través de la sensibilización y el condicionamiento (memoria a corto plazo).

- El aprendizaje entraña diversas formas de plasticidad sináptica para crear formas complejas de conexión.

- La memoria se forma por etapas. La memoria a corto plazo dura unos minutos, esta gradualmente -mediante la repetición- se convierte en memoria a largo plazo y puede durar muchos días o períodos más extensos.

- La memoria a largo plazo no es una mera extensión de la de corto plazo. Con la primera aumenta la cantidad y activación sináptica, con la segunda decrece.

- Con el reforzamiento necesario se producen mejores recuerdos, por tanto, el aprendizaje es duradero.

Si se hace una analogía con el ser humano, los aportes de Kandell son una primera base para comprender los esquemas de aprendizaje vistos como resultado de la experiencia humana. Para estudiarlos, no solo es necesario conocer lo que ocurre en el cerebro (lo que no es objeto de esta tesis), sino pensar que estos procesos se manifiestan en el comportamiento del sujeto. Por tanto, reproducir un determinado aprendizaje, implica un orden de realización complejo, que como bien apuntan Maturana y Dávila (2008) permite observarlo, conocerlo, entenderlo y explicarlo, mediante nuevas formas de percibir la acción humana.

Lo anterior implica que tanto el observado como el observador comparten conocimientos y prácticas. Esto es así, porque los individuos están dotados de un sistema nervioso, entre cuyos 
componentes se encuentra un sistema que favorece el aprendizaje. Es decir, toda experiencia deja una huella inscripta en el cerebro, producto de la plasticidad (Anserment y Magistretti, 2006), pero esa impronta - desde el punto del aprendizaje- no se impresiona de la misma manera en cada sujeto, por tanto, la explicación de Kandell (2007) sobre los mecanismos que lo producen y sus efectos, cuando se trata del aprendizaje humano, se encuentra con una situación compleja.

\subsection{Las neuronas espejo}

Otra teoría que puede ayudar al estudio de los esquemas de aprendizaje es la propuesta por Iacoboni (2009). Este plantea que existe un sistema neuronal, que codifica las intenciones de los demás, es decir además de leer el mundo, los humanos leen a otros humanos. Este sistema está compuesto por grupos de células nerviosas especializadas -neuronas espejo- que permiten entenderlos. Cada persona puede entender el dolor de otra, las acciones, el lenguaje ${ }^{2}$, porque se ha formado en el cerebro una especie de plantilla neuronal, correlato de ese sentimiento, lenguaje o acción.

El sistema de neuronas espejo puede diferenciar las acciones humanas y su intencionalidad en diversos contextos. Esto se debe a que el cerebro puede reflejarlas de manera especular, por tanto, el sujeto puede anticipar los movimientos y palabras de los demás -estas células nerviosas se ubican cerca de la corteza motora primaria, que envía señales eléctricas a los músculos, por ello, cooperan con el comportamiento motor. La experimentación respalda la hipótesis de que las neuronas espejo codifican las intenciones. Se observó mayor actividad de estas mientras los individuos observaban la acción prensil en el contexto de beber que en el de limpiar, debido a que beber es una intención más compleja que limpiar (Iacoboni et al, 2005). Esas mismas regiones con sus células espejo se activan cuando cada sujeto logra las propias intenciones en relación con la acción realizada por los demás (Iacoboni, 2009).

Los gestos son parte esencial del lenguaje, tanto como las palabras, las frases y las oraciones, por lo que conforman un mismo sistema. Los gestos representan en muchas ocasiones lo que con el lenguaje no se consigue (McNeill, 1992). Golding-Meadow (1999), logró observar que la falta de correlación entre palabras y gestos genera una alta actividad en el cerebro que favorece el aprendizaje de conceptos nuevos en estudiantes jóvenes. Alibali, Heath y Myers (2001) estudiaron como la presencia de una pantalla que separaba al hablante del oyente eliminaba la realización de gestos icónicos que reflejan el contenido del discurso y no a los gestos rítmicos que solo marcan movimientos rítmicos de las manos. Los investigadores concluyeron que los primeros ayudan a la comprensión del oyente y los segundos a la expresión del hablante. Por su parte, (Molnar-Szakacs, Wilson y Iacoboni, en Iacoboni, 2009), comprobaron que las neuronas espejo se activan más cuando el individuo observa los gestos icónicos que facilitan la comunicación y la comprensión, que al observar gestos rítmicos.

2. Se excluye las personas autistas sin tratamiento. 
La hipótesis de que las neuronas espejo tienen mayor excitación cuando codifican manipulaciones organizadas jerárquicamente (Greenfield, 1991), es importante para comprender cómo estas células cooperan en la realización y comprensión del lenguaje articulado. Los experimentos de Fadiga, et al (2000) en los cuales se utilizaron dos tipos de palabras -unas con sonidos fuetes de vibrante múltiple y otras con sonidos leves de labio dental fricativas sordasconfirmaron que cuando los individuos escuchaban palabras con movimientos linguales fuertes, estos pequeños movimientos de la lengua realizaron mayor activación de la corteza motora, que los leves. Otro experimento que consistió en pronunciar sílabas en voz alta, mientras se escuchaba con audífonos pronunciarlas a otras personas, confirmó que se activaban la misma área motora cuando hablaban y cuando escuchaban, (Wilson, et al, 2004).

En síntesis, el cerebro-cuerpo humano es un sistema autopoiético, cuya capacidad plástica y el sistema de neuronas espejo pueden producir aprendizaje. No importa el modelo mental que se adopte -hay cuatro generaciones de teorías sobre la mente- estas capacidades son reales en todos los seres vivos. Debido a que en este estudio se ha recurrido a los esquemas de acción, como teoría para investigar sobre el aprendizaje gramatical, se requiere un argumento que al menos ayude a comprender cómo se procesa la información para convertirse en esquemas. Hasta el momento, solo se sabe qué ocurre en las estructuras físicas interneurales, pero no se logra captar esas imágenes, modelos o esquemas, que se sabe son reales, porque conviven con sus dueños, los únicos que pueden confirmar su existencia.

Una teoría importante que se acerca a esto es la holografía de la mente. Un holograma ofrece la visión completa del todo en tres dimensiones, por ello se piensa que esto es lo que ocurre en el cerebro. El modelo holográfico de la mente explica que la consciencia se almacena en todo el cerebro, y que surge cada vez que se requiere utilizar la información. Hace una selección recolectándola de todas partes, mediante una suerte de análisis matemático tempo-espacial y la sintetiza en una sola imagen simbólica (Melo, 2011).

Esta propiedad se descubrió en los centros visuales de ratones, cuando se les extirpaba hasta un $90 \%$ de la corteza visual estos continuaban realizando actividades que requerían el uso de la visión (Briggs y Peat, 2005). Así, el cerebro procesa imágenes mediante un holograma interno, y en cada una de sus partes se reconstruye la totalidad de lo captado por los ojos o cualquier otro de los sentidos. Físicamente, las hondas eléctricas se distribuyen por todo el cerebro producto de que la concentración de células nerviosas es densa. Por ello, al expandirse se entrecruzan entre sí formando colecciones caleidoscópicas de interferencias de información nerviosa, lo cual puede conferirle a este órgano propiedades holográficas (Talbot, 2007). 
Las ideas anteriores se basan en el modelo propuesto por Príbram y Ramírez (1980). Estos lo llamaron holograma nervioso, el cual tiene la siguiente configuración funcional, que trata de explicar en una escala de observación microscópica cómo surge el aprendizaje humano:

- Una red neuronal de tres capas celulares denominadas: de entrada, de transformación y de salida. En esta ocurren configuraciones dinámicas y estáticas, las cuales interactúan entre sí.

- La sensibilidad local de un punto concreto de una célula que mide la aportación efectiva que la neurona presináptica hace a la activación total en dicho lugar.

- La contribución depende del lugar donde llega la información, es decir de su posición y de las microestructuras de las conexiones neuronales.

- Microestructuras celulares donde se almacenaría la información de forma estática.

- Las propiedades resultantes de la actividad interneuronal: almacenamiento, reconocimiento y recuerdo, dependen de la actividad de la citoarquitectura específica de los coeficientes de acoplamiento y de los valores de sensibilidad de las membranas neuronales.

- Para que la red neuronal almacene información requiere que no se pierda en su transformación de entrada.

- Lo que se almacena no es la imagen real y compleja, sino los valores de intensidad de la información.

- La información recibida se distribuirá por todas las redes neuronales, que forman regiones donde se inicia una microestructura sináptica relativamente estable.

En contra de este modelo, se han esbozado varias críticas. De ellas, la más relevante es la dualista ¿quién mira al holograma del cerebro? La respuesta: la emergencia misma de la acción corporizada. Varela, Thompson y Rosch (1997), con un enfoque integrador consideran que las cogniciones humanas ocurren por la experiencia senso-motriz del cuerpo que pertenece a un contexto biológico, psicológico y cultural más amplio. Es decir, el sujeto-mundo está determinado tanto por su actuar como por los acontecimientos ambientales, por ello se puede considerar un ser hologramático.

Como seres vivos hologramáticos cada parte del cuerpo-mundo es un puente entre ambos órdenes de la realidad: identidad individual y la holonomía del todo. Por tanto, se reflejan las partes de la totalidad desde cada individualidad. Esta particularización, se explica, porque todos los sectores del cerebro participan en cualquier representación, respetando las correspondientes especializaciones; por consiguiente, no se limita a ser un conjunto de 
modalidades de procesamiento informacional, sino que ciertas formas de conocimientos están anidadas y distribuidas por todo el cerebro (Melo, 2011).

La distribución de la información no solo es posible por la capacidad autopoiética del organismo, sino que en cierta medida está determinada, también, por el holomovimiento. A decir, es producto de las conexiones no locales del mundo subatómico como base de todas las manifestaciones que existen en el universo material:

(...) el contenido o significado que está «plegado» $y$ «transportado» es, principalmente, un orden y una medida que permiten el desarrollo de una estructura (...) lo que «transporta» un orden implicado es el holomovimiento, que a su vez es también una totalidad no fragmentada ni dividida. En algunos casos podremos abstraer aspectos particulares del holomovimiento (por ejemplo, la luz, los electrones, el sonido, etcétera), pero, más generalmente, todas las formas de holomovimiento se entremezclan y son inseparables. Así, en su totalidad, el holomovimiento no se encuentra limitado de ningún modo en absoluto (Bohm, 1998, p. 122)

La mente, el pensamiento, emociones, deseos, voluntad, etc, que se basan en la memoria, son procesos materiales, manifestaciones que emergen del orden implicado como lo hace toda la materia, por ello, no son diferentes de esta (Bohm y Weber, 1982). Por tanto, son parte de un dominio que no va más allá de la materia y expresa orden, coherencia, unidad y totalidad en el plano físico. "Es un movimiento más profundo y extenso que crea, mantiene y finalmente disuelve la estructura" (Bohm, 2002, p. 129).

Las estructuras que soportan o en las que se manifiesta las ideas de Bohm (1998), Príbram y Ramírez (1980), pueden ser las presentadas por Salatino (2009). Este considera que la corteza cerebral -la mayoría de los neurobiólogos lo acepta- está formada por una doble estructuración anatómico-funcional: la disposición horizontal de capas paralelas a la corteza y la distribución de arreglos verticales en forma de columnas que atraviesan en forma ortogonal a las capas horizontales.

A pesar de la polémica que genera este tipo de teorías, hay suficiente evidencia que sugiere que la disposición anatómica columnar, evoca un patrón citoarquitectónico básico sobre el que se edifica la estructura de la parte funcionalmente más importante del cerebro en la misma especie y en especies diferentes.

La unidad básica de la corteza madura es la minicolumna. Está representada por una cadena de neuronas de disposición vertical que atraviesa desde la capa II hasta la VI (Mountcastle, 1997). En estudios con primates, se logró observar que cada minicolumna, contiene entre 80 y 100 neuronas; excepto en la corteza visual donde el número es 2.5 veces mayor. En humanos, el diámetro de estas estructuras es significativo (de 40 a $50 \mu \mathrm{m}$ ), y están separadas unas de otras, por unos $60 \mu \mathrm{m}$ (Buxhoeveden y Casanova, 2002a). 
Las minicolumnas están reunidas en estructuras mayores, llamadas macrocolumnas o módulos de 300 a $600 \mu \mathrm{m}$ de diámetro. Cada una de estas contiene unas 60 a 80 minicolumnas rodeadas por otras seis columnas. Estas adoptan una disposición aproximadamente hexagonal, con forma de panal de abejas (Favorov y Diamond, 1998a). Esta disposición se ha podido comprobar en varias regiones de la corteza cerebral mediante una serie de indicadores anatómicos y fisiológicos (Buxhoeveden y Casanova, 2002b).

Todos los procesos biológicos operan en el dominio del tiempo, este afecta a las columnas, a sus células, vías de conducción y sinapsis. La secuencia temporal afecta más a la organización vertical de las columnas que a la horizontal. Por ello, el sector horizontal comprendido en cada una, haría que las capas horizontales afecten a todas las fases temporales de la actividad neuronal (Buxhoeveden y Casanova, 2002a). Sin una correcta secuencia en la actividad neural, el sistema nervioso degeneraría en un caos y la interface entre micro y macrocolumna se perdería, por consiguiente, el aprendizaje no ocurriría.

De lo anterior, se extrae que esta organización columnar es la base sobre la que, a manera de correlatos nerviosos, se conforma la información captada del mundo, se almacena y se activa, de acuerdo con las exigencias que el sistema cerebro-corporal humano tenga. Por ello, esta estructura y su funcionamiento puede ser la base de la formación de los esquemas de aprendizaje.

\subsection{La memoria}

Con las consideraciones anteriores, se puede afirmar que el funcionamiento del cerebro es producto de una cantidad increíble de procesos mentales. Estos tienen como base de operación, en un ser humano maduro, el sistema de almacenamiento que guarda no solo la experiencia diaria, sino los recuerdos de las experiencias vividas. Estas son individuales y colectivas, porque el recuerdo -que se entiende como aprendizaje- no solo es propiedad de un individuo, sino de todos aquellos que participan de los hechos sociales que lo rodean. Es decir, estamos en presencia de una memoria colectiva (Halbwachs, 2004).

La memoria humana tiene dos componentes: el sistema de memoria individual y el sistema colectivo. El primer sistema está en el cerebro de cada persona, tiene sus tipos, maneras de funcionar y utilidades. Para ello, es necesario considerar la memoria como parte de la actividad humana, de su actividad social, sus procesos formativos y desarrollo de sus capacidades. En este sentido, se podrían distinguir los siguientes tipos y niveles, desde un modelo que considera el sistema emotivo y social del individuo: 
Cuadro 1. Tipos de memorias basadas en las emociones y en el comportamiento social.

\begin{tabular}{|c|c|}
\hline $\begin{array}{c}\text { MEMORIA DE REPRESENTACIÓN DE } \\
\text { NIVEL CONSCIENTE }\end{array}$ & $\begin{array}{c}\text { MEMORIAS DE PROCEDIMIENTOS DEL } \\
\text { NIVEL CONSCIENTE }\end{array}$ \\
\hline $\begin{array}{c}\text { Memorias de representaciones de nivel } \\
\text { subconsciente }\end{array}$ & $\begin{array}{c}\text { Memorias de procedimientos de nivel } \\
\text { subconsciente }\end{array}$ \\
\hline Representaciones afectivas & Procedimientos emotivos \\
\hline
\end{tabular}

a) Intrapersonales: 1 . En relación con la alegría: dicha, placer, felicidad, éxito, jubilo, entusiasmo, animación, orgullo, optimismo, alivio. 2. En relación con la tristeza: sufrimiento, abatimiento, pena, depresión, nostalgia, abandono, vergüenza, culpa, remordimiento, melancolía, malestar.

b) Interpersonales: 1 . En relación con el amor: cariño, ternura, estima, deseo sexual, lujuria, orgasmo. 2. En relación con la cólera: agravio, frustración, indignación, desprecio, envidia, celos, odio, ira, venganza, disgusto.

c) Extra personales: 1. En relación con la sorpresa: admiración, incredulidad, estupor, éxtasis, asco. 2. En relación con la angustia: alarma, susto, horror, terror, tensión, preocupación, pavor, pánico.

\begin{tabular}{|c|c|}
\hline Representaciones cognitivas & Procedimientos productivos \\
\hline $\begin{array}{l}\text { - Respecto del espacio personal (corporal): es- } \\
\text { quema corporal. } \\
\text { - Respecto del espacio peri personal (el espacio } \\
\text { que nos rodea y está al alcance de las manos): } \\
\text { comprende el conocimiento de las relaciones } \\
\text { espaciales de las cosas entre sí y en relación } \\
\text { con uno mismo -como distancia, perspecti- } \\
\text { va, ubicación, tamaño, forma, peso, textura, } \\
\text { humedad; melodía, armonía, ritmo, léxico; } \\
\text { uso de objetos e instrumentos; conocimiento } \\
\text { de rostros y de otras personas. }\end{array}$ & $\begin{array}{l}\text { - Respecto del espacio telepersonal: de rutas, } \\
\text { lugares, orientación en el espacio exterior; } \\
\text { el conocimiento empírico y científico del } \\
\text { mundo exterior, que incluye las cosas y pro- } \\
\text { cesos no experimentados por uno mismo. }\end{array}$ \\
\hline Representaciones conativas & Procedimientos volitivos \\
\hline $\begin{array}{l}\text { - Motivos fundamentales: convicciones y res- } \\
\text { ponsabilidades. }\end{array}$ & $\begin{array}{l}\text { Motivos operativos del trabajo social: } 1 \text {. in- } \\
\text { tenciones, obligaciones y deberes; } 2 \text {. aspira- } \\
\text { ciones, intereses, objetivos y propósitos; } 3 . \\
\text { prejuicios, perspectivas y pretensiones; } 4 \text {. } \\
\text { Ideales y pasiones. }\end{array}$ \\
\hline
\end{tabular}


Otra clasificación sobre la memoria la considera como un sistema de procesamiento de información, que crea el sentido a partir de una serie de procesos necesarios que dan como resultado un ser humano que enfrenta la realidad desde ese saber que ha creado:

Cuadro 2. Tipos de memorias como sistema de procesamiento de información.

\begin{tabular}{|c|c|c|c|}
\hline \multicolumn{2}{|c|}{$\begin{array}{l}\text { MEMORIA DE REPRESENTACIÓN DE } \\
\text { NIVEL CONSCIENTE }\end{array}$} & \multicolumn{2}{|c|}{$\begin{array}{l}\text { MEMORIAS DE PROCEDIMIENTOS DEL } \\
\text { NIVEL CONSCIENTE }\end{array}$} \\
\hline \multicolumn{2}{|c|}{$\begin{array}{c}\text { Memorias de representaciones de nivel } \\
\text { subconsciente }\end{array}$} & \multicolumn{2}{|c|}{$\begin{array}{l}\text { Memorias de procedimientos de nivel } \\
\text { subconsciente }\end{array}$} \\
\hline \multicolumn{2}{|c|}{ Representaciones afectivas } & \multicolumn{2}{|c|}{ Procedimientos emotivos } \\
\hline \multicolumn{4}{|c|}{$\begin{array}{l}\text { a) Memoria episódica, de hechos y eventos con una localización espacial y temporal específica } \\
\text { que incluye, por ejemplo, la información autobiográfica. } \\
\text { b) Memoria semántica, contiene el conocimiento enciclopédico adquirido por medio de } \\
\text { la cultura y la educación (como son) el significado de las palabras, el cálculo, la información } \\
\text { geográfica, la histórica, etc. } \\
\text { c) Memoria de trabajo, almacenamiento temporal de la información que se usa para guiar } \\
\text { una acción futura. }\end{array}$} \\
\hline $\begin{array}{l}\text { Representaciones } \\
\text { perceptuales }\end{array}$ & $\begin{array}{r}\text { Represe } \\
\text { imagi }\end{array}$ & $\begin{array}{l}\text { Procedimientos } \\
\text { conceptuales }\end{array}$ & $\begin{array}{l}\text { Procedimientos de } \\
\text { actuación }\end{array}$ \\
\hline $\begin{array}{l}\text { Son efecto de un } \\
\text { proceso de aprecia- } \\
\text { ción, confrontación, } \\
\text { valoración y uso de } \\
\text { la información res- } \\
\text { pecto de situaciones } \\
\text { intrapersonales, pe- } \\
\text { ripersonales y ex- } \\
\text { trapersonales. }\end{array}$ & $\begin{array}{l}\text { Esta memoria de } \\
\text { imágenes nos pro- } \\
\text { porciona informa- } \\
\text { ción autobiográfica, } \\
\text { pues reproduce la } \\
\text { categoría de datos } \\
\text { que corresponden a } \\
\text { la propia experien- } \\
\text { cia. }\end{array}$ & $\begin{array}{l}\text { Los conceptos se } \\
\text { comprenden y usan } \\
\text { mejor una vez co- } \\
\text { dificados en el len- } \\
\text { guaje hablado, o } \\
\text { algún otro afín. }\end{array}$ & $\begin{array}{l}\text { En el nivel epicons- } \\
\text { ciente se integran } \\
\text { disposiciones, ap- } \\
\text { titudes y actitudes } \\
\text { para constituir el } \\
\text { plano de la actua- } \\
\text { ción. }\end{array}$ \\
\hline & & & \\
\hline
\end{tabular}




\begin{tabular}{|c|c|c|c|}
\hline $\begin{array}{l}\text { La retención de in- } \\
\text { formación sensorial } \\
\text {-afectiva o cogniti- } \\
\text { va- para su procesa- } \\
\text { miento perceptual, } \\
\text { requiere de una red } \\
\text { neural distribuida e } \\
\text { interconectada en } \\
\text { paralelo en ambos } \\
\text { hemisferios cere- } \\
\text { brales, cuya activi- } \\
\text { dad debe mantener- } \\
\text { se todo el tiempo } \\
\text { necesario para su } \\
\text { confrontación cons- } \\
\text { ciente. }\end{array}$ & $\begin{array}{l}\text { - Información res- } \\
\text { pecto de los acon- } \\
\text { tecimientos en que } \\
\text { toma parte uno } \\
\text { mismo, y respecto } \\
\text { de las cosas que uno } \\
\text { manipula o usa di- } \\
\text { recta y prácticamen- } \\
\text { te. Se trata de viven- } \\
\text { cias y experiencias } \\
\text { que reflejan hechos } \\
\text { realizados por uno } \\
\text { mismo, o cosas he- } \\
\text { chas o experimenta- } \\
\text { das por uno mismo. }\end{array}$ & $\begin{array}{l}\text { Se ha supuesto } \\
\text { que el habla es el } \\
\text { contenido real de } \\
\text { la memoria expli- } \\
\text { cita -sobre todo } \\
\text { semántica- y se lo } \\
\text { considera inheren- } \\
\text { te al pensamiento } \\
\text { abstracto y su im- } \\
\text { portancia en los } \\
\text { lenguajes sociales } \\
\text {-el habla, la lógica, } \\
\text { la matemática, la } \\
\text { computación. }\end{array}$ & $\begin{array}{l}\text { - Se organiza el pro- } \\
\text { ceso productivo (o } \\
\text { creativo), la pro- } \\
\text { ducción psíquica } \\
\text { de tipo cognitivo se } \\
\text { integra dentro del } \\
\text { plan de actuación } \\
\text { anticipado (men- } \\
\text { tal) que es el mode- } \\
\text { lo que organiza la } \\
\text { actividad personal } \\
\text { que se realiza en la } \\
\text { ejecución practica } \\
\text { en la forma de con- } \\
\text { ducta, desempeño } \\
\text { o comportamiento } \\
\text { efectivo u objetual } \\
\text { de la personalidad } \\
\text { en tanto agente } \\
\text { que influye sobre } \\
\text { los demás o sobre } \\
\text { las cosas. }\end{array}$ \\
\hline
\end{tabular}

Fuente: (Adaptado de Ortiz, 1998, pp. 49-61)

Los tipos de memoria individual abordados funcionan, según la utilidad que el o los sujetos les den. Sin embargo, tanto la capacidad de memoria como la de atención tienen límites. Esta capacidad está asociada con el tiempo y cantidad de información que se retiene y requiere para el funcionamiento del ser humano, por ello, Ortiz, (1998) expresa que:

Después de que la información -cuyos datos están codificados en los sistemas de memoria en el nivel subconsciente- se recupera, reconstruye o accede al plano epiconsciente, su utilización como modelo de la actividad personal actual requiere de la retención de dicha información en este nivel de la actividad consciente y su permanencia en uno de sus planos de la percepción, la imaginación, el pensamiento o la actuación, (p. 58).

Por consiguiente, la idea de la limitación que tiene el cerebro para mantener la información activa, no es del todo válida para los recuerdos de larga duración. En el curso de una vida humana media, el individuo puede almacenar 2,8 x $10^{20}$ bits de información (Talbot, 2007). A decir, la capacidad de acumulación de información en relación con la capacidad de activación son diferentes, pero funcionan de manera concatenada. 
Otro aspecto importante que se debe destacar es el 'proceso de consolidación', con el cual la información procesada se vuelve recuerdo en niveles cada vez más profundos de almacenamiento. Esto es así, para todas las funciones neuropsicológicas y con toda la información, porque las neuronas literalmente crecen y se conectan juntas (Hebb, 2002), generando primero cambios puramente fisiológicos -las sinapsis del circuito se vuelven más permeables al mismo estímuloy luego cambios anatómicos permanentes. Por lo tanto, la activación de las sinapsis moviliza en las células mecanismos genéticos que causan más sinapsis en esas uniones axónicas (Solms y Turnbull, 2004).

Los tipos de memoria dan al ser humano una gran capacidad para manejar el mundo complejo que lo rodea. Así, reconoce personas, lugares, imagina acontecimientos, resuelve problemas, hipotetiza sobre el futuro, hace vida social, porque razona, aprende y recuerda lo que tiene engramatizado en su cerebro (Damasio, 2007, 2010), pero a la vez, utiliza en tiempo real la memoria externa que también ha construido. A esta Bartra (2007) la denomina exocerebro, este dispositivo de corte ambiental y social está compuesto por circuitos exocerebrales simbólicos que apoyan y amplían la capacidad de los circuitos cerebrales: memorias artificiales (bibliotecas, memorias USB, discos duros de PC, museos), engramas artístico-icónicos (pinturas, esculturas, edificios, mapas, planos), registros de sonido (discos de acetato, CD, DVD, en los que se graba la imagen y voz humana, además de otros sonidos), y finalmente, el grupo social, como exocerebro vivo, que evoca los recuerdos, el conocimiento como un todo, mediante el lenguaje.

El lenguaje, función cerebral de alto nivel, sustenta diversas capacidades mentales generales: crear y manipular símbolos, combinar conceptos y categorías, procesar información auditiva y visual a gran velocidad, recuperar información de la memoria a largo plazo (MLP) y mantener activa en la memoria de trabajo estructuras de saber complejo (Demestre, 2003). Lo anterior ocurre, porque la activación de información, por el uso del lenguaje, correlacionada desde la MLP es un proceso automatizado que ocurre durante la comprensión. La memoria a corto plazo (MCP) transmite señales en paralelo a los contenidos de la MLP, la cual se activa mediante un proceso 'pasivo' de resonancia neuronal, sobre un determinado elemento por la sobreposición de caracteres semánticos y la fuerza de asociación entre un elemento activado y un indicio que viaja o se distribuye por la fuerza de activación de la red neuronal (Kintsch, 1988; McKoon y Ratcliff, 1992; Mckoon et al, 1996, Myers y O’Brien, 1998, en Fernández, 2002).

La manera en que funciona el cerebro, y en especial, la memoria tiene implicaciones sobre el proceso de aprendizaje. Bruning, Schraw, Norby y Ronning (2005) señalan como relevantes, aquellas en las que el docente debe hacer sus consideraciones: 
- El procesamiento de la información está limitado por la memoria sensorial y a corto plazo, por tanto, los estudiantes requieren dirigir sus recursos selectivamente a la información relevante.

- La automatización, mediante la práctica extensiva, facilita el aprendizaje reduciendo las limitaciones de los recursos para completar las tareas.

- La percepción y la atención están guiadas por el conocimiento previo, que se activa mediante estímulos que ayudan a rescatarlos de la memoria.

- La percepción y la atención son procesos flexibles y automáticos que se pueden mejorar frente a cada tarea.

- Tanto las limitaciones de los recursos como los datos limitan el aprendizaje e influyen en la resolución de las tareas.

- Se debe animar a gestionar los recursos de cada individuo, enseñándoles a autorregular su conocimiento, sus estrategias y motivación.

- El procesamiento de la información es más fácil cuando se reparte en la memoria de trabajo lo que hay que aprender.

Por tanto, es en el trabajo mente-cuerpo (sistema de memoria) donde se forman los esquemas de acción de cada uno de los individuos. Estos no solo se almacenan en el cerebro, sino también se amplían al exocerebro contextual. Además, se puede afirmar que se activan por la acción del individuo que piensa, se mueve y sobre todo habla para comunicarse con los demás.

Esto alienta a considerar el cuidado que se debe tener no solo con la instrumentación didáctica, sino con la acción y el lenguaje que se utiliza en un aula de clase. Tanto el docente como los estudiantes tienen sus propias intencionalidades -entendidas por Searle (2004) como la capacidad de la mente para referirse o versar sobre o corresponderse con objetos y situaciones del mundo al margen de sí mismos-, a las cuales hacen referencia mediante lo que dicen, comprenden y maneras de realizar sus acciones en las situaciones de aprendizaje.

\section{CONCLUSIONES}

Los planteamientos teóricos arriba desarrollados favorecen, o antes bien crean un modelo de interpretación conceptual que permite adentrarse en los desarrollos teóricos experimentales sobre los esquemas de acción, o como los denominamos: los esquemas de aprendizaje humanos. Por ello, se puede concluir que: 
- El sistema cerebral tiene una capacidad intrínseca denominada plasticidad cerebral, mediante la cual se reestructura a sí mismo en una escala de la realidad, imperceptible para los sentidos humanos.

- Esta propiedad es real y causalmente es producida por el funcionamiento del sistema nervioso, los efectos del ambiente sobre el organismo vivo y la experiencia de este en relación con las situaciones a las que se enfrenta.

- Existe un sistema neuronal espejo que codifica las intenciones de los demás: "los humanos leen a otros humanos", por ello, permite entendernos.

- Los esquemas de aprendizaje se pueden estructurar, considerando el modelo holográfico de la mente. Este explica que la consciencia (el ser y su experiencia) se almacena en todo el cerebro, y surge cada vez que se requiere utilizar la información. Esto lo hace seleccionándola y recolectándola de todas partes, mediante una suerte de análisis matemático tempo-espacial y la sintetiza en una sola imagen simbólica: el esquema mental.

- El funcionamiento del cerebro es producto de una cantidad increíble de procesos mentales. Estos tienen como base de operación, en un ser humano maduro, el sistema de almacenamiento que guarda no solo la experiencia diaria, sino los recuerdos de las experiencias vividas. Este sistema se denomina memoria individual y memoria colectiva. En estas memorias el recuerdo -entendido como aprendizaje- no solo es propiedad de un individuo, sino de todos aquellos que participan de los hechos sociales que lo rodean.

- Las memorias dan al ser humano una gran capacidad para manejar el mundo complejo que lo rodea. Así, reconoce personas, lugares, imagina acontecimientos, resuelve problemas, hipotetiza sobre el futuro, hace vida social, porque razona, aprende y recuerda lo que tiene engramatizado en su cerebro.

- Finalmente, el lenguaje sustenta diversas capacidades mentales generales: crear y manipular símbolos, combinar conceptos y categorías, procesar información auditiva y visual a gran velocidad, recuperar información de la memoria a largo plazo (MLP) y mantener activa en la memoria de trabajo estructuras de saber complejo. Es decir, esta función es la que permite la manifestación comunicativa de los esquemas mentales, a través de la interacción con los demás. 


\section{REFERENCIAS BIBLIOGRÁFICAS}

Alibali, M. Heath, D. y Myers, H. (2001). Effects of visibility between speaker and listener on gesture production: Some gestures are mean to be seen. Journal of Memory and Language, 44.pp. 169-188.

Ansermet, F. y Magistretti, P. (2006). A cada cual su cerebro. Plasticidad neuronal $e$ inconsciente. Madrid: Katz.

Barta, R. (2007). Antropología del cerebro. La conciencia y los sistemas simbólicos. México D.F: Fondo de Cultura Económica.

Bohm, D. (1998). La totalidad y el orden implicado. ( $3^{\mathrm{a}}$ ed). Barcelona: Kairós.

Bohm, D. (2002). Sobre la creatividad. Barcelona: Kairós.

Bohm, D. y Weber, R. (1982). El universo no manifestado y el universo manifestado. En Krishnamurti, J. (1982). Dentro de la mente. Barcelona: Sirio.

Bruning, R. Schraw, G. Norby, M. y Ronning, R. (2005). Psicología cognitiva y de la instrucción. Madrid: Pearson.

Buxhoeveden, D. P.; Casanova, M. F. (2002a). The minicolumn hipótesis. In NeuroscienceBrain, vol, 125, pp. 935-951. Recuperado el 15 de febrero de 2014 de http://brain. oxfordjournals.org/content/125/5/935. full.pdf + html.

Buxhoeveden, D. P.; Casanova, M. F. (2002b). The Minicolumn and Evolution of the Brain.Brain Behav, vol, 60, pp. 125-151.
Damasio, A. (2007). El error de Descartes. (4 ed.). Barcelona: Drakontos Bolsillo.

Damasio, A. (2010). Y el cerebro creó al hombre. ( $2^{\mathrm{a}}$ ed.). Barcelona: Desino.

De la Torre, E. y Navarro, R. (1982) Metodología de la investigación bibliográfica, archivística y documental, es la base de la ciencia. MacGraw-Hill: México.

Demestre, J. (2003). Procesamiento de frases e información léxica. (Tesis de Doctorado). Tarragona: Universitat Rovira i Virgili.

Fadiga, L. et al. (2000). Speach, listening specifically modulates the excitability of tongue muscles: A TMS study". EuropeanJournal of Neuroscience, vol, 15. pp. 399-402.

Favorov, OV. y Diamond, ME. (1988a). Demonstration of discrete place-defined columns - segregates - in the cat SI. J Comp Neurol 1990; 298: 1988a; 472: 2542.

Fernández, S. (2002). Factores sintácticos y semánticos en el procesamiento del lenguaje. (Tesis de Doctorado). Madrid: Universidad Complutense de Madrid.

Golding-Meadow, S. (1999). The rol of gesture in comunication and thinking. Trends in CognitiveScience, vol, 3.pp. 419-429.

Greenfield, P. (1991). Language, tools and brain: The ontogeny and philogeny of hierarquically organized sequential 
behavior. Behavior, BrainScience, vol, 14.pp. 531-595.

Halbwachs, M. (2004). La memoria colectiva. Zaragoza: Universidad de Zaragoza.

Hebb, D. O. (2002). The organization of Behavior. A neuropsycological therory. Lawrence Erlbaum Associates

Iacoboni, M. (2009). Las neuronas espejo. Empatía, neuropolítica, autismo, imitación, o de cómo entendemos a los otros. Madrid: Katz.

Iacoboni, M. et al (2005). Grasping the intentions of others with one's own mirror neuron system. PLos Biology, vol, 3. p.79.

Kandell. E. (2007). En busca de la memoria. El nacimiento de una nueva ciencia de la mente. (Trad. Marengo. E.). Madrid: Conocimiento).

Luzoro, J. (2006). Humberto Maturana. Desde la biología a la psicología. Chile: Editorial Universitaria.

Maturana, H. (1990). Biología de la cognición y la epistemología. Chile: Universidad de la Frontera.

Maturana, H. y Dávila, X. (2008). Habitar lo humano. En seis ensayos de biología cultural. Chile: Comunicaciones del Noreste.

Maturana, H. y Pörksen, B. (2004). Del ser al hacer. Los orígenes de la biología del conocer. Chile: Comunicaciones del Noreste.

Maturana, H. y Varela, F. (2003). El árbol del conocimiento. Las bases bilógicas del entendimiento humano. Buenos Aires, Argentina: Lumen.

McNeill, D. (1992). Hand and mind: Whar gesture reveal about thougth. Chicago: University of Chicago Press.

Melo, A. (2011). Cerebro, mente y conciencia. Un enfoque multidisciplinario. Barcelona: iMedPub.

Mountcastle, V. B. (1997). The columnar organization of the neocortex-Brain, 120, pp.701-722.Recuperado el 15 de febrero de 2014 de http://brain.oxfordjournals. org/content/120/4/701.full.pdf+htm.

Ortiz, P. (1998). El nivel consciente de la memoria. Una hipótesis de trabajo. Lima: Fondo de Desarrollo Editorial de la Universidad de Lima.

Príbram, K. y Ramírez, J. (1980). Cerebro, mente y holograma. Madrid: Alhambra.

Purves, et.al. (2007). Neurociencia. (3 ${ }^{\mathrm{a}}$. ed). México: Panamericana.

Redondo, I. (2012). Un modelo experiencialista de la cognición aplicado al estudio filosófico de la ciencia. (Tesis de Doctorado): Universitat Autònoma de Barcelona. Recuperado el 25 de febrero de 2013 de http://www. tesisenred.net/handle/10803/107946.

Salatino, D. (2009). Semiótica de los sistemas reales. Análisis semiótico de la emergencia psico-bio-socio-cultural. (Tesis de Doctorado). Mendoza, Argentina: Universidad de Cuyo.Recuperado el 15 de marzo de 2013 de http://www.academia. 
edu/778540/SEMI\%C3\%93TICA_DE_ LOS_SISTEMAS_REALES.

Searle, J. (2004). Mente, lenguaje y sociedad. La filosofía del mundo real. Madrid: Alianza Editorial.

Sierra, Bravo. R. (1986). Tesis doctorales y trabajos de investigación científica. Thomson: Madrid.

Solms, M. y Turnbull, O. (2004). El cerebro $y$ el mundo interior. Una introducción a la neurociencia de la experiencia subjetiva. Bogotá: Fondo de Cultura Económica.

Talbot. M. (2007). El universo holográfico. Una visión nueva y extraordinaria de la realidad. (Trad. Del Yerro, C.). Madrid: Palmyra.
Varela, F. Thompson, E. y Rosch. E. (1997). De cuerpo presente. Las ciencias cognitivas y la experiencia humana. Barcelona: Gedisa.

Wilson, S. et al. (2004). Listening to speech activates motor areas involved in speach production. Nature Neuroscience, vol, 7. En Iacoboni, M. (2009). Las neuronas espejo. Empatía, neuropolítica, autismo, imitación, o de cómo entendemos a los otros. Madrid: Katz. 